\title{
First-in-human trial of an anti-5T4 antibody-monomethylauristatin conjugate, PF-06263507, in patients with advanced solid tumors
}

\author{
Geoffrey I. Shapiro ${ }^{1}$ - Ulka N. Vaishampayan ${ }^{2} \cdot$ Patricia LoRusso $^{3} \cdot$ Jeremy Barton $^{4}$. \\ Steven Hua ${ }^{4}$ Steven D. Reich ${ }^{4}$. Ronald Shazer ${ }^{4}$ Carrie T. Taylor ${ }^{4,5}$ • Dawei Xuan ${ }^{4}$. \\ Hossein Borghaei ${ }^{6}$
}

Received: 16 November 2016/Accepted: 22 December 2016/Published online: 9 January 2017

(C) The Author(s) 2017. This article is published with open access at Springerlink.com

Summary Background The antibody-drug conjugate PF06263507 targets the cell-surface, tumor-associated antigen $5 \mathrm{~T} 4$ and consists of a humanized IgG1 conjugated to the microtubule-disrupting agent monomethylauristatin- $\mathrm{F}$ by a non-cleavable maleimidocaproyl linker. In this first-in-human, dose-finding trial (NCT01891669), we evaluated safety, pharmacokinetics, and preliminary antitumor activity of PF06263507 in pretreated patients with advanced solid tumors, unselected for $5 \mathrm{~T} 4$ expression. starting at $0.05 \mathrm{mg} / \mathrm{kg}$, with 25 , 56 , and $95 \%$ dose increments, depending on observed doselimiting toxicities (DLTs), applying a modified continual reassessment method. Results Twenty-six patients received PF06263507 at 0.05 to $6.5 \mathrm{mg} / \mathrm{kg}$. The first DLT, grade 3 photophobia, occurred at $4.34 \mathrm{mg} / \mathrm{kg}$ and two additional DLTs, grade 2 keratitis and grade 1 limbal stem cell deficiency $(>2$ week dosing delay), at $6.5 \mathrm{mg} / \mathrm{kg}$. The most common adverse events (AEs) were fatigue (38.5\%), photophobia (26.9\%), and decreased appetite, dry eye, nausea, and thrombocytopenia

Geoffrey I. Shapiro

geoffrey_shapiro@dfci.harvard.edu

$\triangle$ Carrie T. Taylor

Carrie.Taylor@pfizer.com

1 Early Drug Development Center, Department of Medical Oncology, Dana-Farber Cancer Institute, 450 Brookline Ave, Mayer 446, Boston, MA 02215, USA

2 Karmanos Cancer Institute, Detroit, MI, USA

3 Yale University, New Haven, CT, USA

4 Pfizer Oncology, La Jolla, CA, USA

5 Pfizer Early Oncology Development and Clinical Research, 10777 Science Center Drive, CB-1, San Diego, CA 92121, USA

6 Fox Chase Cancer Center, Philadelphia, PA, USA
(23.1\% each). No treatment-related grade 4-5 AEs were reported. Systemic exposure of PF-06263507 increased in a dose-related manner. At the maximum tolerated dose (MTD, $4.34 \mathrm{mg} / \mathrm{kg}$ ), mean terminal half-life for PF-06263507 and unconjugated payload were $\sim 6$ and 3 days, respectively. Payload serum concentrations were substantially lower compared with PF-06263507. No objective responses were observed. Conclusions The MTD and recommended phase II dose were determined to be $4.34 \mathrm{mg} / \mathrm{kg}$. Ocular toxicities accounted for the DLTs observed, as previously reported with monomethylauristatin-F payloads. Further studies are warranted to investigate clinical activity of this agent in patients with 5T4-expressing tumors.

Trial registration ID: NCT01891669

Keywords 5T4 $\cdot$ PF-06263507 $\cdot$ Solid tumors ·

Monomethylauristatin conjugate $\cdot$ Immunoconjugate .

Antibody-drug conjugate

\section{Introduction}

Antibody-drug conjugates (ADCs) were developed to improve the therapeutic index of cytotoxic anti-cancer agents. ADCs consist of immunoconjugates in which a cytotoxic agent is chemically linked to an antibody that selectively binds to an internalizing tumor-associated antigen. This approach allows delivery of the cytotoxic agent to the tumor while minimizing exposure of normal tissues $[1,2]$.

5T4, also known as trophoblast glycoprotein, is a cell surface antigen that is rapidly internalized [3, 4]. Expression of 5T4, as defined by immunohistochemistry, has been observed in a variety of solid tumors (i.e., lung, breast, ovarian, endometrial, bladder, pancreatic, esophageal, and gastric cancers), 
whereas expression in normal, adult tissues was found to be limited [5-11]. 5T4 expression has been associated with advanced disease and/or worse clinical outcomes in patients with non-small-cell lung, colorectal, ovarian, or gastric cancer and pre-B acute lymphoblastic leukemia [7, 9-12].

PF-06263507 (5T4 ADC) is an ADC comprised of the humanized anti-5T4 IgG1 antibody PF-06281192 (huA1 $\mathrm{mAb}$ ) conjugated via cysteine (Cys) residues to the microtubule-disrupting agent monomethylauristatin $\mathrm{F}$ (MMAF) by a maleimidocaproyl (mc) linker, at an average drug:antibody molecular ratio of 4:1 (Fig. 1) [2, 13-15]. PF-06281192 recognizes a conformational epitope on the extracellular domain of human and cynomolgus monkey 5T4. MMAF is an auristatin, a fully synthetic, pentapeptide inhibitor of tubulin polymerization that ultimately induces $\mathrm{G} 2 /$ mitosis cell cycle arrest and cell death at low picomolar intracellular concentrations. Cys-capped mc linker plus MMAF (Cys-mcMMAF, PF06264490) constitutes the released active moiety following catabolism in the lysosome of an ADC with an mc linker to MMAF. Results from in vitro studies showed that MMAF and Cys-mcMMAF inhibited tubulin polymerization at equivalent doses, suggesting that they have comparable intracellular activity [13]. PF-06263507 was developed for the treatment of adult patients with advanced solid tumors expressing $5 \mathrm{~T} 4$.

In vitro, the 5T4 ADC PF-06263507 and huA1 mAb PF-06281192 showed specific binding to tumor cells expressing the 5T4 antigen and rapid internalization [15]. In cell proliferation assays, PF-06263507 mediated cytotoxicity against cultured tumor cells in a 5T4-dependent manner and inhibition of tumor spheres growth in 3dimensional culture. In preclinical studies in vivo, PF06263507 demonstrated potent anti-tumor activity against a panel of human tumor xenografts (i.e., lung and breast cancer) with low, moderate, and high 5T4 expression levels. In contrast, treatment with the unconjugated antibody (PF-06281192) or a control ADC did not inhibit tumor growth [15].

This first-in-human, dose-finding, phase I study was designed to evaluate the safety, tolerability, pharmacokinetics (PK), and preliminary antitumor activity of PF-06263507 in patients with advanced solid malignancies.

\section{Methods}

\section{Study design}

This was a phase I, open-label, multi-center, single arm, doseescalation study (NCT01891669) of single-agent PF06263507 in sequential cohorts of adult patients with advanced solid tumors for whom no standard therapy was available; tumor 5T4 expression was not required for eligibility, based on the lack of availability of a CLIA-certified assay and because of the widespread expression of the antigen on the surface of multiple tumor types. Based on prior toxicity studies conducted in cynomolgus monkeys and rats (unpublished data), showing toxic effects potentially related to the Cys-mcMMAF payload on the cardiovascular system (e.g., myocardial degeneration/necrosis and/or fibrosis, premature ventricular contractions, vasculopathy), liver (e.g., multifocal sinusoidal ectasia, atrophy of hepatocytes), kidney (e.g., glomerulonephropathy and/or degeneration/regeneration of tubular epithelium), and the hematologic system (e.g., thrombocytopenia, anemia), the study protocol specified methods for assessing and monitoring potential adverse effects of PF06263507 on these systems, including administration of initial doses in an inpatient facility to closely monitor treated patients.

The primary objective was to evaluate safety and tolerability at increasing doses of PF-06263507, determine the maximum tolerated dose (MTD) and select the recommended phase II dose (RP2D). Secondary objectives were to evaluate the overall safety profile; characterize single- and multipledose PK of PF-06263507, PF-06281192, and unconjugated payload (Cys-mcMMAF, PF-06264490); evaluate the immunogenicity of PF-06263507; and document any preliminary evidence of anti-tumor activity. The modified continual reassessment method (mCRM) algorithm [16, 17] was utilized to determine the MTD and run at the end of each cohort to determine whether the dose of PF-06263507 should be escalated, re-visited, or de-escalated, based on cumulative toxicity data from patients in the ongoing and all previous cohorts (see Supplemental Information for further method details).

The study protocol was approved by each participating center's Institutional Review Board and written informed consent was obtained from each patient. The study was conducted

Fig. 1 Structure of PF-06263507

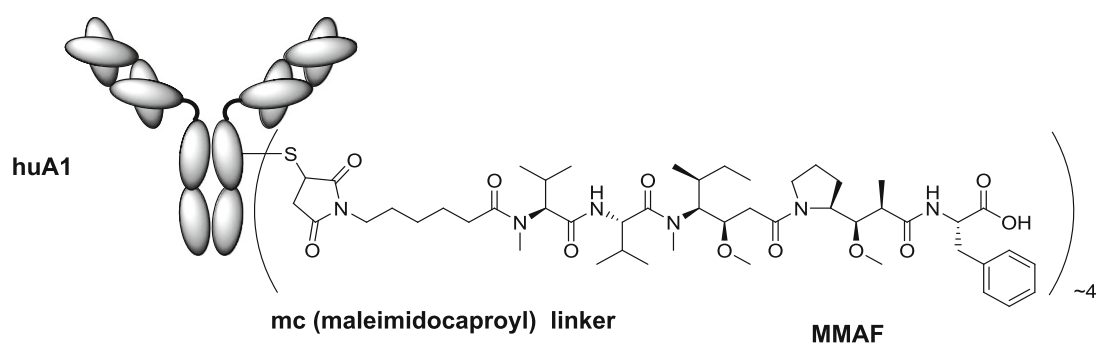


in compliance with the Declaration of Helsinki and followed the International Congress of Harmonisation Good Clinical Practices guidelines.

\section{Patient entry criteria}

Adult patients were included in this study if they had histological or cytological diagnosis of locally advanced or metastatic solid tumors unresponsive to treatment or with no available standard therapy; Eastern Cooperative Oncology Group (ECOG) performance status $0-1$; and adequate bone marrow, renal, liver, and cardiac function. Patients were excluded if they had symptomatic or untreated brain metastases; had received major surgery, radiation treatment, or systemic anti-cancer therapy within 4 weeks prior to study entry; had previously experienced a significant allergic reaction to recombinant human or murine proteins; or had an active and clinically significant bacterial, fungal, or viral infection (i.e., hepatitis B, hepatic $\mathrm{C}$, or immunodeficiency virus infection).

\section{Treatment and DLT}

PF-06263507 was administered on day 1 of each 21-day cycle as an intravenous infusion over approximately $60 \mathrm{~min}$, on an inpatient basis. Patients received PF-06263507 until disease progression, unacceptable toxicity, withdrawal of consent, or study termination. No premedication was required. Dose interruption during dosing, dose modification in the following cycle, or discontinuation were allowed by protocol in patients experiencing DLT. Intra-patient dose escalation was not permitted.

During dose escalation any of the following AEs occurring in the first 21-day treatment period and attributable to PF-06263507 were classified as DLT: a) hematologic: grade 4 neutropenia lasting $>7$ days; febrile neutropenia (grade 3 neutropenia and a single body temperature $>38.3{ }^{\circ} \mathrm{C}$ or a sustained temperature of $38{ }^{\circ} \mathrm{C}$ for $>$ $1 \mathrm{~h}$ ); grade $\geq 3$ neutropenia with infection; any-grade thrombocytopenia associated with clinically significant or life-threatening bleeding; grade 4 thrombocytopenia; b) non-hematologic: maximally treated grade $\geq 3$ AEs; a confirmed positive cardiac troponin I result ( $>$ 99th percentile); delay by $>2$ weeks in receiving the next scheduled treatment cycle due to persisting toxicities attributable to PF-06263507. In addition, clinically important or persisting grade 2 AEs could be considered a DLT by the investigators and the study sponsor. Grade $\geq 3$ cytokine release syndrome, infusion reaction, and allergic reaction were not to be considered DLTs, but could be a reason for patient discontinuation from the study.

\section{Safety and efficacy assessments}

Baseline evaluations, including complete blood counts, serum chemistries, vital signs, and 12-lead electrocardiograms, were performed within 4 weeks prior to the start of treatment. A follow-up visit 28-35 days after treatment discontinuation was required to monitor for any AEs. AEs were graded using the NCI CTCAE version 4.03 [18]. Computed tomography (CT) or magnetic resonance imaging (MRI) scans were obtained within 4 weeks prior to start of treatment and every 6 weeks until disease progression or end of treatment. Efficacy was assessed by Response Evaluation Criteria in Solid Tumors (RECIST) 1.1 [19].

\section{PK and immunogenicity assessments}

Serial blood samples for PK analysis of PF-06263507, PF06281192, and PF-06264490 were collected at multiple time points during cycles 1 and 4, pre-dose for all other cycles, and at the end of treatment. Serum concentrations of PF-06263507 and PF-06281192 were measured using validated enzymelinked immunosorbent assays. Serum concentrations of unconjugated payload were measured with a validated liquid chromatography and tandem mass spectrometry assay. The limits of quantitation for PF-06263507, PF-06281192, and PF-06264490 were 20,35 , and $0.05 \mathrm{ng} / \mathrm{mL}$, respectively. PK parameters for PF-06263507, PF-06281192, and PF06264490 following intravenous infusion of PF-06263507 were calculated by non-compartmental analysis using an internally validated electronic non-compartmental analysis software (eNCA) version 2.2.4. Samples below the lower limit of quantification were set to 0 for analysis.

Immunogenicity of PF-06263507 was assessed using validated electrochemiluminescence assays, with blood samples collected pre-dose on days 1 and 15 of cycle 1, pre-dose in every cycle thereafter, and at the end of treatment. Samples positive for anti-PF-06263507 (anti-drug) antibodies (ADA) were also analyzed for neutralizing antibodies.

\section{Results}

\section{Patient characteristics}

Twenty-seven patients were enrolled; 1 patient died of disease progression prior to start of study treatment. Twenty-six patients received treatment with PF-06263507 at doses ranging from 0.05 to $6.5 \mathrm{mg} / \mathrm{kg}$, between 21 August 2013 and 11 March 2015. Patients (58\% women) were 25-88 years old and had been previously treated with a median of 6 (range 1-13) prior regimens. There were no meaningful differences among the treatment dose cohorts in the reported demographic or baseline patient characteristics (Table 1). Primary cancer 
Table 1 Patient demographics and baseline characteristics

PF-06263507 (mg/kg)

\begin{tabular}{llllllllll}
\hline 0.05 & 0.10 & 0.19 & 0.37 & 0.73 & 1.42 & 2.78 & 4.34 & 5.42 & 6.5 \\
$n=2$ & $n=2$ & $n=2$ & $n=2$ & $n=2$ & $n=2$ & $n=2$ & $n=6$ & $n=3$
\end{tabular}

\begin{tabular}{|c|c|c|c|c|c|c|c|c|c|c|}
\hline \multicolumn{11}{|l|}{ Gender, $\mathrm{n}$} \\
\hline Male & 1 & 1 & 1 & 2 & 1 & 0 & 0 & 2 & 1 & 2 \\
\hline Female & 1 & 1 & 1 & 0 & 1 & 2 & 2 & 4 & 2 & 1 \\
\hline \multicolumn{11}{|l|}{ Age (years) } \\
\hline Mean & 60.5 & 57.0 & 41.0 & 60.0 & 53.5 & 62.0 & 61.5 & 65.2 & 57.7 & 67.7 \\
\hline Range & $59-62$ & $54-60$ & $25-57$ & $55-65$ & $43-64$ & $36-88$ & $56-67$ & $58-85$ & $52-62$ & $61-72$ \\
\hline \multicolumn{11}{|l|}{ Race, $\mathrm{n} \%$} \\
\hline White & $2(100)$ & $1(50)$ & $2(100)$ & $2(100)$ & $2(100)$ & $2(100)$ & $2(100)$ & $6(100)$ & $3(100)$ & $2(67)$ \\
\hline Black & 0 & $1(50)$ & 0 & 0 & 0 & 0 & 0 & 0 & 0 & $1(33)$ \\
\hline \multicolumn{11}{|l|}{ Ethnicity, n \% } \\
\hline Hispanic/Latino & 0 & 0 & 0 & 0 & 0 & 0 & 0 & 0 & 0 & $1(33)$ \\
\hline Not Hispanic/Latino & $2(100)$ & $2(100)$ & $2(100)$ & $2(100)$ & $2(100)$ & $2(100)$ & $2(100)$ & $6(100)$ & $3(100)$ & $2(67)$ \\
\hline \multicolumn{11}{|l|}{ ECOG performance status, $\mathrm{n} \%$} \\
\hline 0 & 0 & 0 & 0 & $2(100)$ & 0 & 0 & 0 & $1(17)$ & 0 & 0 \\
\hline 1 & $2(100)$ & $2(100)$ & $2(100)$ & 0 & $2(100)$ & $2(100)$ & $2(100)$ & $5(83)$ & $3(100)$ & $3(100)$ \\
\hline \multicolumn{11}{|l|}{ Cancer diagnosis, $\mathrm{n} \%$} \\
\hline Colorectal cancer & $1(50)$ & $1(50)$ & 0 & $1(50)$ & $1(50)$ & 0 & $1(50)$ & 0 & 0 & 0 \\
\hline Ovarian cancer & 0 & 0 & 0 & 0 & 0 & $1(50)$ & 0 & $1(17)$ & $1(33)$ & $1(33)$ \\
\hline Lung cancer & 0 & 0 & $1(50)$ & $1(50)$ & 0 & 0 & 0 & $1(17)$ & 0 & 0 \\
\hline Cholangiocarcinoma & 0 & 0 & 0 & 0 & 0 & 0 & 0 & $1(17)$ & 0 & $1(33)$ \\
\hline Hepatocellular carcinoma & 0 & 0 & $1(50)$ & 0 & 0 & 0 & 0 & $1(17)$ & 0 & 0 \\
\hline Pancreatic cancer & 0 & 0 & 0 & 0 & $1(50)$ & 0 & 0 & 0 & 0 & $1(33)$ \\
\hline Other* & $1(50)^{\mathrm{a}}$ & $1(50)^{b}$ & 0 & 0 & 0 & $1(50)^{\mathrm{c}}$ & $1(50)^{d}$ & $2(33)^{e f}$ & $2(67)^{g h}$ & 0 \\
\hline
\end{tabular}

ECOG Eastern Cooperative Oncology Group

* Other cancers included 1 patient each with ${ }^{a}$ renal cell carcinoma, ${ }^{b}$ cervical cancer, ${ }^{\mathrm{c}}$ bladder cancer, ${ }^{\mathrm{d}}$ esophageal cancer, ${ }^{\mathrm{e}}$ adenoid cystic carcinoma, ${ }^{\mathrm{f}}$ mesothelioma, ${ }^{\mathrm{g}}$ adenoma of unknown primary, and ${ }^{\mathrm{h}}$ breast cancer

diagnoses included colorectal cancer $(n=5)$, ovarian cancer $(n=4)$, lung cancer $(n=3)$, and cholangiocarcinoma, hepatocellular carcinoma, and pancreatic cancer ( $n=2$ each). Eight patients had different tumor types, as listed in Table 1.

\section{Dose assessment and DLT}

Two patients each were enrolled at the PF-06263507 starting dose of $0.05 \mathrm{mg} / \mathrm{kg}$ and at increasing doses $(0.10,0.19,0.37$, $0.73,1.42 \mathrm{mg} / \mathrm{kg}$ ) up to $2.78 \mathrm{mg} / \mathrm{kg}$ with no DLTs observed. A DLT of grade 3 photophobia occurred on day 15 of cycle 1 at the $4.34 \mathrm{mg} / \mathrm{kg}$ dose level. This cohort was expanded to 6 patients and no additional DLTs were observed. Three patients treated at the $5.42 \mathrm{mg} / \mathrm{kg}$ dose level did not experience DLT. Of the 3 patients enrolled in the subsequent $6.5 \mathrm{mg} / \mathrm{kg}$ dose cohort, 1 patient had a DLT of grade 2 keratitis on day 9 of cycle 1 and 1 patient had grade 1 limbal stem cell deficiency associated with blurred vision and photophobia, at the first visit after cycle 1 . As the latter patient was not retreated with
PF-06263507, this constituted a treatment delay of $>2$ weeks and met the definition of DLT.

The investigators and the study sponsor determined that at the $6.5 \mathrm{mg} / \mathrm{kg}$ dose level the MTD had been exceeded. One DLT at the $6.5 \mathrm{mg} / \mathrm{kg}$ dose would have led to dose de-escalation to $5.42 \mathrm{mg} / \mathrm{kg}$ and 2 DLTs in 3 patients at $6.5 \mathrm{mg} / \mathrm{kg}$ to dose deescalation to $4.34 \mathrm{mg} / \mathrm{kg}$, as per study design based on the mCRM algorithm (see also Supplemental Information). The MTD was determined to be $4.34 \mathrm{mg} / \mathrm{kg}$ based on the DLTs observed, the number of patients $(n=6)$ treated at this dose, and other AEs noted throughout dose escalation. At the estimated MTD, DLT occurred in 1 of 6 patients (17\%).

\section{Safety profile}

Of the 26 patients evaluable for safety, all experienced $\geq 1$ treatment-emergent $\mathrm{AE}$ and $22(84.6 \%)$ had $\geq 1$ treatmentrelated AEs. The most frequently reported all causality AEs were fatigue $(46.2 \%)$, decreased appetite $(38.5 \%)$, nausea 
(30.8\%), vomiting (30.8\%), photophobia (26.9\%), abdominal distension $(23.1 \%)$, increased aspartate aminotransferase $(23.1 \%)$, dry eye $(23.1 \%)$, thrombocytopenia $(23.1 \%)$, cough $(19.2 \%)$, dyspnea $(19.2 \%)$, peripheral edema (19.2\%), and blurred vision $(19.2 \%)$.

The most frequently observed all-grade, treatment-related AEs were fatigue (38.5\%), photophobia (26.9\%), decreased appetite $(23.1 \%)$, dry eye $(23.1 \%)$, nausea $(23.1 \%)$, thrombocytopenia $(23.1 \%)$, and vomiting $(19.2 \%)$ (Table 2$)$. At the MTD $(n=6)$, fatigue $(n=4)$, nausea $(n=3)$, and photophobia $(n=3)$ were the treatment-related AEs observed in more than 2 patients.

All causality and treatment-related grade 3-4 AEs reported are summarized in Table 3 by dose group. Treatment-related grade 3 AEs occurred in 3 patients at the higher dose levels, including: eye pain, photophobia, and inflammation $(n=1$; $4.34 \mathrm{mg} / \mathrm{kg}$ ); increased alanine aminotransferase and increased blood alkaline phosphatase $(n=1 ; 5.42 \mathrm{mg} / \mathrm{kg})$; and thrombocytopenia $(n=1,6.5 \mathrm{mg} / \mathrm{kg}$ ). One patient in the $2.78 \mathrm{mg} / \mathrm{kg}$ group had a grade $4 \mathrm{AE}$ of hypercalcemia, which was not considered treatment-related. One non-treatment related death due to exacerbation of chronic obstructive pulmonary disease occurred in a patient in the $4.34 \mathrm{mg} / \mathrm{kg}$ group within 28 days of last treatment dose. No grade 4-5 treatment-related AEs were observed in patients receiving PF-06263507.
Although thrombocytopenia had been observed in the animal toxicity studies, only 1 patient $(6.5 \mathrm{mg} / \mathrm{kg}$ group $)$ developed grade 3 thrombocytopenia, on day 8 of cycle 1 , which represented the nadir for this patient. Four patients had grade 2 thrombocytopenia including 2 patients each at 4.34 and $6.5 \mathrm{mg} / \mathrm{kg}$. The nadir appeared to occur on day 8 of cycle 1 , with recovery above $75 \times 10^{9} / \mathrm{L}$ by day 15 of cycle 1 .

In addition to the patient who developed an inflammatory syndrome on day 9 and grade 3 photophobia and eye pain on day $15,10(38.5 \%)$ patients had grade 1-2 treatment-related ocular AEs. All patients had undergone eye examinations as part of the general physical examination prior to the first dose of study drug, and no clinically significant abnormalities were found. Grade 1-2 treatment-emergent AEs occurring in $>1$ patient were photophobia $(23.1 \%)$; dry eye, or dry eye syndrome (23.1\%); eye pain (11.5\%); blurred vision (11.5\%); and conjunctivitis, increased lacrimation, and vitreous floaters (each 7.7\%) (Table 2). These AEs were observed as early as day 1 , but usually by day 15 , and as late as day 52 of treatment. The patient with inflammatory syndrome and conjunctivitis was treated with erythromycin ointment and ophthalmic prednisolone acetate, with no changes in PF-06263507 administration.

Treatment-related AEs led to study-drug discontinuation in 3 patients: $1(4.34 \mathrm{mg} / \mathrm{kg})$ due to photophobia; a second $(6.5 \mathrm{mg} / \mathrm{kg})$ due to bilateral annular keratitis, and the third
Table 2 Treatment-related adverse events reported in 2 or more patients

\begin{tabular}{llllll}
\hline AE & $\begin{array}{l}\text { Grade } 1 \\
\mathrm{n}(\%)\end{array}$ & $\begin{array}{l}\text { Grade } 2 \\
\mathrm{n}(\%)\end{array}$ & $\begin{array}{l}\text { Grade } 3 \\
\mathrm{n}(\%)\end{array}$ & $\begin{array}{l}\text { Grade } 4 \\
\mathrm{n}(\%)\end{array}$ & $\begin{array}{l}\text { Total } \\
\mathrm{n}(\%)\end{array}$ \\
\hline Any AE & $15(57.7)$ & $4(15.4)$ & $3(11.5)$ & 0 & $22(84.6)$ \\
Fatigue & $9(34.6)$ & $1(3.8)$ & 0 & 0 & $10(38.5)$ \\
Photophobia & $5(19.2)$ & $1(3.8)$ & $1(3.8)$ & 0 & $7(26.9)$ \\
Decreased appetite & $5(19.2)$ & $1(3.8)$ & 0 & 0 & $6(23.1)$ \\
Dry eye & $4(15.4)$ & $2(7.7)$ & 0 & 0 & $6(23.1)$ \\
Nausea & $4(15.4)$ & $2(7.7)$ & 0 & 0 & $6(23.1)$ \\
Thrombocytopenia & $2(7.7)$ & $3(11.5)$ & $1(3.8)$ & 0 & $6(23.1)$ \\
Vomiting & $3(11.5)$ & $2(7.7)$ & 0 & 0 & $5(19.2)$ \\
Eye pain & $3(11.5)$ & 0 & $1(3.8)$ & 0 & $4(15.4)$ \\
Increased AST & 0 & $2(7.7)$ & $1(3.8)$ & 0 & $3(11.5)$ \\
Headache & $3(11.5)$ & 0 & 0 & 0 & $3(11.5)$ \\
Blurred vision & $1(3.8)$ & $2(7.7)$ & 0 & 0 & $3(11.5)$ \\
Increased ALT & $2(7.7)$ & 0 & 0 & 0 & $2(7.7)$ \\
Anemia & $1(3.8)$ & $1(3.8)$ & 0 & 0 & $2(7.7)$ \\
Conjunctivitis & 0 & $2(7.7)$ & 0 & 0 & $2(7.7)$ \\
Dysgeusia & $1(3.8)$ & $1(3.8)$ & 0 & 0 & $2(7.7)$ \\
Increased lacrimation & $2(7.7)$ & 0 & 0 & 0 & $2(7.7)$ \\
Peripheral sensory neuropathy & $2(7.7)$ & 0 & 0 & 0 & $2(7.7)$ \\
Vitreous floaters & $2(7.7)$ & 0 & 0 & 0 & $2(7.7)$ \\
\hline
\end{tabular}

$A E$ adverse event, $A L T$ alanine aminotransferase, $A S T$ aspartate aminotransferase 
Table 3 All causality and treatment-related grade 3-4 adverse events

\begin{tabular}{|c|c|c|c|c|c|c|c|c|c|c|}
\hline \multirow[t]{3}{*}{$\begin{array}{l}\mathrm{AE} \\
\mathrm{n}, \%\end{array}$} & \multicolumn{10}{|c|}{$\begin{array}{l}\mathrm{PF}-06263507 \\
\mathrm{mg} / \mathrm{kg}\end{array}$} \\
\hline & \multicolumn{2}{|l|}{$\begin{array}{l}0.1 \\
n=2\end{array}$} & \multicolumn{2}{|l|}{$\begin{array}{l}2.78 \\
n=2\end{array}$} & \multicolumn{2}{|l|}{$\begin{array}{l}4.34 \\
n=6\end{array}$} & \multicolumn{2}{|l|}{$\begin{array}{l}5.42 \\
n=3\end{array}$} & \multicolumn{2}{|l|}{$\begin{array}{l}6.5 \\
n=3\end{array}$} \\
\hline & All & Related & All & Related & All & Related & All & Related & All & Related \\
\hline Any AEs & $1(50)$ & 0 & $1(50)$ & 0 & $3(50)$ & $1(16.7)$ & $1(33.3)$ & $1(33.3)$ & $2(66.7)$ & $1(33.3)$ \\
\hline Thrombocytopenia & 0 & 0 & 0 & 0 & 0 & 0 & 0 & 0 & $1(33.3)$ & $1(33.3)$ \\
\hline Eye pain & 0 & 0 & 0 & 0 & $1(16.7)$ & $1(16.7)$ & 0 & 0 & 0 & 0 \\
\hline Photophobia & 0 & 0 & 0 & 0 & $1(16.7)$ & $1(16.7)$ & 0 & 0 & 0 & 0 \\
\hline Inflammation & 0 & 0 & 0 & 0 & $1(16.7)$ & $1(16.7)$ & 0 & 0 & 0 & 0 \\
\hline Hepatobiliary disease & 0 & 0 & 0 & 0 & 0 & 0 & 0 & 0 & $1(33.3)$ & 0 \\
\hline Device-related infection & 0 & 0 & 0 & 0 & $1(16.7)$ & 0 & 0 & 0 & 0 & 0 \\
\hline Increased AST & 0 & 0 & 0 & 0 & 0 & 0 & $1(33.3)$ & $1(33.3)$ & 0 & 0 \\
\hline Increased blood alkaline phosphatase & 0 & 0 & 0 & 0 & 0 & 0 & $1(33.3)$ & $1(33.3)$ & 0 & 0 \\
\hline Hypercalcemia & 0 & 0 & $1(50)$ & 0 & 0 & 0 & 0 & 0 & 0 & 0 \\
\hline Hyponatremia & $1(50.0)$ & 0 & 0 & 0 & 0 & 0 & 0 & 0 & 0 & 0 \\
\hline Hypophosphatemia & 0 & 0 & 0 & 0 & $1(16.7)$ & 0 & 0 & 0 & 0 & 0 \\
\hline Embolism & 0 & 0 & 0 & 0 & $1(16.7)$ & 0 & 0 & 0 & 0 & 0 \\
\hline
\end{tabular}

$A E$ adverse event, $A S T$ aspartate aminotransferase; related, treatment-related

one $(6.5 \mathrm{mg} / \mathrm{kg})$ due to limbal stem cell deficiency. Both grade 3 photophobia and grade 2 keratitis resolved without sequelae.

\section{Anti-tumor activity}

No objective responses were observed in this study. Two patients achieved stable disease (at the lowest and the highest dose of PF-06263507), 19 patients had disease progression as best response and 2 experienced symptomatic deterioration. Best response was undetermined in 3 patients because of $\mathrm{AE}$, death, or withdrawal from the study.

\section{Pharmacokinetics}

The cycle 1 PK parameters for PF-06263507 (5T4 ADC) are summarized in Table 4. PK exposures for PF-06263507 generally increased in a dose-related manner across the $0.05-$ $6.5 \mathrm{mg} / \mathrm{kg}$ dose range. The cycle $1 \mathrm{PK}$ parameters were best characterized at the $4.34 \mathrm{mg} / \mathrm{kg}$ dose level, where data were available from 6 patients. At $4.34 \mathrm{mg} / \mathrm{kg}$, mean clearance for PF-06263507 was $\sim 0.7 \mathrm{~L} /$ day, the mean volume of distribution $\left(\mathrm{V}_{\mathrm{ss}}\right)$ was estimated to be $5.3 \mathrm{~L}$, approximately the physiologic blood volume and similar to that of human IgG antibodies, and the mean terminal half-life $\left(\mathrm{t}_{1 / 2}\right)$ was $\sim 6$ days.

Cycle 1 mean serum concentration-time profiles for PF06263507, PF-06281192, and PF-06264490 following a single intravenous infusion of PF-06263507 $4.34 \mathrm{mg} / \mathrm{kg}$ are presented in Fig. 2. PF-06281192 (total antibody) concentrationtime profiles generally resembled those of PF-06263507 at the
$4.34 \mathrm{mg} / \mathrm{kg}$ dose, but with longer terminal $t_{1 / 2}$ values of $\sim 8.8$ days. PF-06264490 (payload) serum concentrations were substantially lower compared to those observed for PF06263507 and PF-06281192 following a $4.34 \mathrm{mg} / \mathrm{kg}$ intravenous dose of PF-06263507. PF-06264490 concentrations appeared to increase following PF-06263507 infusion and reached a mean maximum concentration $\left(\mathrm{C}_{\max }\right)$ of $3 \mathrm{ng} / \mathrm{mL}$ at $\sim 8 \mathrm{~h}$ post-dose. PF-06264490 mean terminal $\mathrm{t}_{1 / 2}$ was 3.4 days, shorter than that observed for PF-06263507.

\section{Immunogenicity}

Two (9\%) patients tested positive for ADA at baseline (day 1, cycle 1). Of the 23 patients who were evaluated for ADA postbaseline, $4(17 \%)$ patients were positive for ADA for at least 1 post-baseline measurement. Four (17\%) patients tested positive for post-baseline neutralizing antibodies. The effect of ADA on PF-06263507 PK was not evaluated due to the small number of patients in each dose group.

\section{Discussion}

We report here findings from the first-in-human trial of the 5T4 ADC PF-06263507 in patients with locally advanced or metastatic solid tumors with no available standard therapy following multiple lines of prior anticancer treatment. 
Table 4 First-dose pharmacokinetic parameters for $\mathrm{PF}-06263507^{\mathrm{a}}$

\begin{tabular}{lllllll}
\hline $\begin{array}{l}\text { Dose } \\
(\mathrm{mg} / \mathrm{kg})\end{array}$ & $\mathrm{N}$ & $\mathrm{C}_{\max }(\mu \mathrm{g} / \mathrm{mL})$ & $\begin{array}{l}\mathrm{AUC}_{\text {inf }} \\
(\mu \mathrm{\mu g} \times \mathrm{d} / \mathrm{mL})\end{array}$ & $\begin{array}{l}\text { Terminal } \mathrm{t}_{1 / 2} \\
(\text { days })\end{array}$ & $\mathrm{V}_{\text {ss }}(\mathrm{L})$ & $\mathrm{CL}(\mathrm{L} /$ day $)$ \\
\hline 0.05 & 2 & $0.8(-)$ & $1.8(-)$ & $3.0(-)$ & $9.5(-)$ & 3.4 \\
0.10 & 2 & $2.4(-)$ & $4.7(-)$ & $1.5(-)$ & $3.5(-)$ & 1.7 \\
0.19 & 2 & $4.0(-)$ & $10.4(-)$ & $4.5(-)$ & $6.2(-)$ & 1.5 \\
0.37 & 2 & $9.5(-)$ & $24.2(-)$ & $3.5(-)$ & $6.3(-)$ & 1.5 \\
0.73 & 2 & $21.2(-)$ & $52.3(-)$ & $4.4(-)$ & $5.0(-)$ & 1.0 \\
1.42 & 2 & $45.5(-)$ & $90.4(-)$ & $3.8(-)$ & $5.5(-)$ & 1.3 \\
2.78 & 2 & $65.7(-)$ & $123.7(-)$ & $4.2(-)$ & $4.2(-)$ & 0.9 \\
4.34 & 6 & $102.9(22 \%)$ & $418.3(12 \%)$ & $6.0(15 \%)$ & $5.3(16 \%)$ & $0.7(22 \%)$ \\
5.42 & 3 & $142.0(48 \%)$ & $332.7(19 \%)$ & $5.4(9 \%)$ & $5.6(21 \%)$ & $1.0(23 \%)$ \\
6.50 & 3 & $109.1(40 \%)$ & $486.2(-)$ & $5.5(-)$ & $7.4(-)$ & 1.1 \\
\hline
\end{tabular}

$C_{\max }$ maximum concentration, $A U C_{i n f}$ area under the curve from 0 to infinity, $t_{1 / 2}$ terminal half-life, $V_{S S}$ volume of distribution at steady state, $C L$ clearance

${ }^{a}$ Data are presented as geometric mean (\% coefficient of variation), with the exception of $t_{1 / 2}$, which is presented as arithmetic mean (\% coefficient of variation). Summary statistics are not presented if fewer than three patients had reportable parameter values
PK exposures for PF-06263507 generally increased in a dose-related manner across the $0.05-6.5 \mathrm{mg} / \mathrm{kg}$ dose range tested. At the estimated MTD $(4.34 \mathrm{mg} / \mathrm{kg})$, the mean terminal half-life for PF-06263507 and unconjugated payload were approximately 6 and 3 days, respectively. The stability of this non-cleavable mc linker is demonstrated by the $\sim 148$-fold difference between molar AUCs of PF-06263507 and unconjugated payload observed in this study.

Patients received the first dose of PF-06263507 on an inpatient basis to allow close observation for potential toxicities. Preclinical findings of toxicity on cardiovascular, hepatic, renal, and hematologic functions (unpublished data) did not translate to clinically significant toxicities at the doses evaluated in this clinical study. PF06263507 demonstrated a favorable safety profile with 1
DLT occurring at the estimated MTD (4.34 mg/kg), no DLTs at $5.42 \mathrm{mg} / \mathrm{kg}$, and 2 DLTs at $6.5 \mathrm{mg} / \mathrm{kg}$. The most frequent, treatment-related AEs at the MTD were fatigue, nausea, and photophobia. Although thrombocytopenia (mostly grade 2) was observed in some patients at the higher doses of PF-06263507 administered in this study, it did not appear to represent a significant safety concern. Furthermore, none of the patients had a $>60 \mathrm{msec}$ increase from baseline in QTcF or a QTcF $\geq 500 \mathrm{msec}$, and there was no evidence of an effect of PF-06263507 on other ECG parameters (data not shown). No treatmentrelated grade 4-5 AEs were reported across all evaluated dose levels of PF-06263507.

Thus, overall, the non-clinical toxicity studies were not predictive of the safety profile observed for PF-06263507
Fig. 2 Mean serum concentration-time profiles (semi$\log$ scale) of PF-06263507, PF06281192 , and PF-06264490 following a single $4.34 \mathrm{mg} / \mathrm{kg}$ intravenous infusion of PF06263507 (cycle 1)

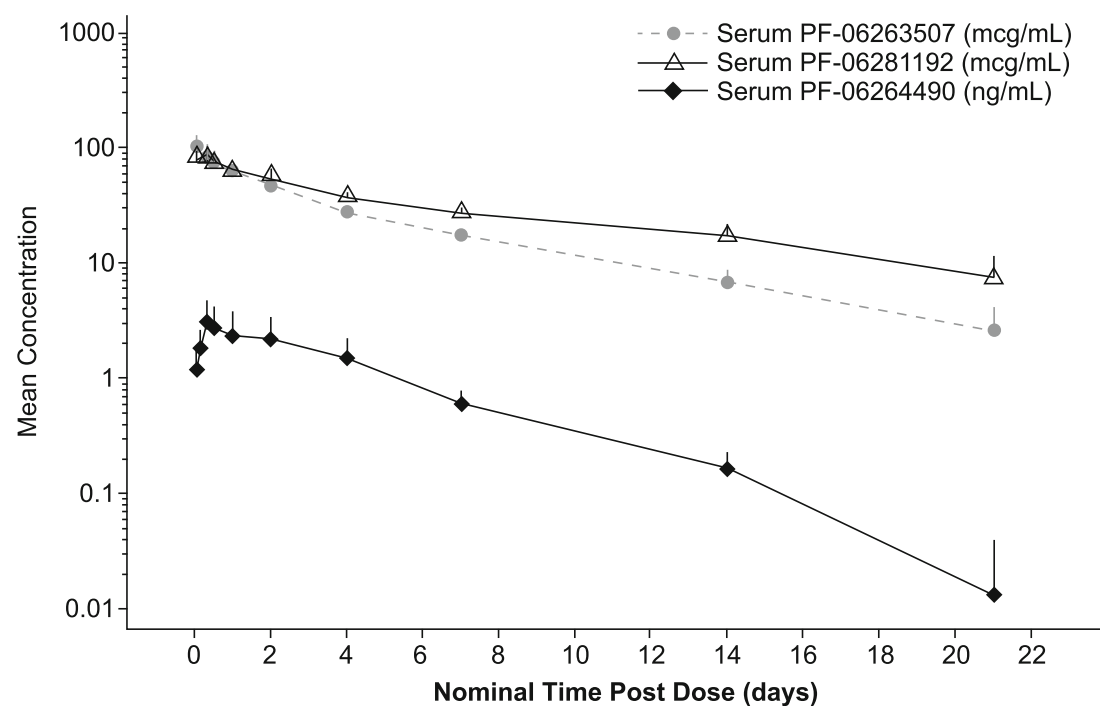


in these cohorts of patients with advanced solid malignancies. However, the ocular AEs noted in this study, although not observed in previous animal safety studies conducted in cynomolgus monkeys and rats (unpublished data), were not unexpected, as they had been previously described in patients following administration of the CD70-targeted ADC SGN-75 and the CD19-targeted ADC SGN-CD19A. Both these ADCs comprise an MMAF payload [20-23]. As reported in 47 patients treated once every 3 weeks with SGN-75, ocular AEs (i.e., corneal epitheliopathy, dry eye) were observed in $57 \%$ of patients [20]. As in the SGN-75 and SGN-CD19A trials, patients with ocular AEs in our study were treated with artificial tears or eye drops containing steroids. Once the ocular AEs were identified, no prophylactic treatment was given to patients as the frequency and severity of these events, especially at lower doses, did not seem to warrant such therapy.

We did not observe any objective responses following treatment with PF-06263507. However, some patients may have received sub-therapeutic doses at the lower dose levels. In addition, 5T4 tumor expression was not required and not determined for the patients included in this phase I study; thus, it is possible that a number of patients had low or no 5T4 tumor expression, which could account for the lack of antitumor activity observed. A companion assay to detect 5T4 expression may, in the future, help to identify patients with the 5T4 expression levels required for PF-06263507 antitumor activity. Recent findings with the folate receptor alpha $(F R \alpha)$-targeting ADC IMGN853 (mirvetuximab soravtansine) in patients with platinum-resistant epithelial ovarian cancer and with the delta-like protein 3 (DLL3)targeted ADC SC16LD6.5 (rovalpituzumab tesirine) in patients with recurrent/refractory small-cell lung cancer indicate that expression levels of the target tumor-associated antigens may represent useful biomarkers for patient selection in ADCbased therapy $[24,25]$.

In addition to patient selection for tumor target expression, combination strategies are of key importance in the development of new, more effective anticancer treatment regimens and should therefore also be considered for ADC-based therapeutic approaches. PF-06263507 could represent an interesting agent for combination with different standard-of-care agents in view of its safety profile; preclinical studies have demonstrated strong synergistic or additive activity of PF-06263507 in combination with gedatolisib (PF-05212384, a pan class I isoform PI3K and mTORC1/2 inhibitor) or taxanes [15, 26-28]. Treatment with PF-06263507 plus gedatolisib resulted in induction of apoptosis and cell line-specific inhibition of the downstream biomarkers pAKT and glycogen synthase kinase. In human tumor xenografts models, dual targeting with a combination of PF-06263507 plus gedatolisib or paclitaxel produced substantially greater antitumor activity and longer survival compared with single-agent treatments, suggesting a critical role of the auristatin payload in the observed synergy. Furthermore, induction by the payloads of potentially immunogenic cell death in treated tumors [15] suggests a rationale for combining ADCbased therapy with immune checkpoint inhibitors.

The field of ADCs is rapidly expanding with currently more than 50 ADCs at different stages of clinical development. Of note, the majority of these ADCs employ a cleavable linker to attach an antibody to a tubulin-targeted payload. Cleavable linkers are associated with a "bystander effect," whereby cleavage of the linker results in the release of a membrane-permeable, active toxin, which mediates killing of target-positive cells, but also induction of "bystander" death in neighboring, nonantigen-expressing cells. PF-06263507 was selected to comprise a 5T4 antibody linked to MMAF via a noncleavable mc linker, as this particular construct showed an improved therapeutic potential compared with several other conjugates that used cleavable auristatins or other payloads $[2,29]$. A further reduction in off-target toxicity may be achieved with antibody-drug conjugates generated by drug conjugation to genetically encoded antibody sites (site-specific conjugation), which minimizes heterogeneity, increases stability, and enhances PK properties and overall efficacy of the immunoconjugates [30].

In conclusion, the 5T4 ADC PF-06263507 was generally well tolerated at the estimated MTD of $4.34 \mathrm{mg} / \mathrm{kg}$ on a once-every-3-week schedule. At higher doses, ocular toxicities were dose-limiting. The RP2D for future studies of PF-06263507 in patients selected for 5T4 tumor expression is $4.34 \mathrm{mg} / \mathrm{kg}$.

Acknowledgements The authors thank all participating patients, their families and caregivers, as well as the global network of investigators, research nurses, study coordinators, and operations staff. This study was supported by Pfizer. Medical writing and editorial support was provided by $\mathrm{S}$. Mariani, MD, PhD, of Engage Scientific Solutions and was funded by Pfizer.

\section{Compliance with ethical standards}

Conflict of interest G. I. Shapiro received research funding from Pfizer. U. N. Vaishampayan has nothing to disclose related to this study. P. LoRusso received an advisory board honorarium from Pfizer. $\mathrm{H}$. Borghaei received research funding from Takeda (Millennium), Merck, and Celgene; and consulting honoraria from BMS, Lilly, Genentech, Pfizer, Celgene, Boehringer-Ingelheim, Trovogene, and EMD-Serono. J. Barton, S. Hua S, R. Shazer R, C. T. Taylor, and D. Xuan were employees of Pfizer during the conduct of this study. S. D. Reich is a contractor for Pfizer.

Research involving human participants All procedures performed in this study involving human participants were in accordance with the ethical standards of the institutional and/or national research committee 
and with the 1964 Helsinki declaration and its later amendments or comparable ethical standards.

Informed consent Informed consent was obtained from all individual participants included in the study.

Open Access This article is distributed under the terms of the Creative Commons Attribution 4.0 International License (http:// creativecommons.org/licenses/by/4.0/), which permits unrestricted use, distribution, and reproduction in any medium, provided you give appropriate credit to the original author(s) and the source, provide a link to the Creative Commons license, and indicate if changes were made.

\section{References}

1. Alley SC, Okeley NM, Senter PD (2010) Antibody-drug conjugates: targeted drug delivery for cancer. Curr Opin Chem Biol 14: 529-537

2. Sapra P, Hooper AT, O'Donnell CJ et al (2011) Investigational antibody drug conjugates for solid tumors. Expert Opin Investig Drugs 20:1131-1149

3. Boghaert ER, Sridharan L, Khandke KM et al (2008) The oncofetal protein, 5T4, is a suitable target for antibody-guided anti-cancer chemotherapy with calicheamicin. Int J Oncol 32:221-234

4. Hole N, Stern PL (1990) Isolation and characterization of 5T4, a tumour-associated antigen. Int J Cancer 45:179-184

5. Hole N, Stern PL (1988) A $72 \mathrm{kD}$ trophoblast glycoprotein defined by a monoclonal antibody. Br J Cancer 57:239-246

6. Southall PJ, Boxer GM, Bagshawe KD et al (1990) Immunohistological distribution of 5T4 antigen in normal and malignant tissues. Br J Cancer 61:89-95

7. Damelin M, Geles KG, Follettie MT et al (2011) Delineation of a cellular hierarchy in lung cancer reveals an oncofetal antigen expressed on tumor-initiating cells. Cancer Res 71:4236-4246

8. Al-Taei S, Salimu J, Lester JF et al (2012) Overexpression and potential targeting of the oncofoetal antigen 5T4 in malignant pleural mesothelioma. Lung Cancer 77:312-318

9. Starzynska T, Marsh PJ, Schofield PF et al (1994) Prognostic significance of 5T4 oncofetal antigen expression in colorectal carcinoma. Br J Cancer 69:899-902

10. Wrigley E, McGown AT, Rennison J et al (1995) 5T4 oncofetal antigen expression in ovarian carcinoma. Int $\mathrm{J}$ Gynecol Cancer 5: 269-274

11. Naganuma H, Kono K, Mori Y et al (2002) Oncofetal antigen 5T4 expression as a prognostic factor in patients with gastric cancer. Anticancer Res 22:1033-1038

12. Castro FV, McGinn OJ, Krishnan S et al (2012) 5 T4 oncofoetal antigen is expressed in high risk of relapse childhood pre-B acute lymphoblastic leukemia and is associated with a more invasive and chemotactic phenotype. Leukemia 26:1487-1498

13. Doronina SO, Mendelsohn BA, Bovee TD et al (2006) Enhanced activity of monomethylauristatin $\mathrm{F}$ through monoclonal antibody delivery: effects of linker technology on efficacy and toxicity. Bioconjug Chem 17:114-124

14. Smith LM, Nesterova A, Alley SC, Torgov MY, Carter PY (2006) Potent cytotoxicity of an auristatin-containing antibody-drug conjugate targeting melanoma cells expressing melanotransferrin/p97. Mol Cancer Ther 5:1474-1482
15. Sapra P, Damelin M, Dijoseph J et al (2013) Long-term tumor regression induced by an antibody-drug conjugate that targets $5 \mathrm{~T} 4$, an oncofetal antigen expressed on tumor-initiating cells. Mol Cancer Ther 12:38-47

16. Goodman SN, Zahurak ML, Piantadosi S (1995) Some practical improvements in the continual reassessment method for phase I studies. Stat Med 4:1149-1161

17. O'Quigley J, Pepe M, Fisher L (1990) Continual reassessment method. A practical design for phase 1 clinical trials in cancer. Biometrics 46:33-48

18. U.S. Department of Health and Human Services, National Cancer Institute. CTCAE v4.03. NIH Publication No. 09-5410, revised June 2010. http://evs.nci.nih.gov/ftp1/CTCAE/CTCAE 4.03 201006-14_QuickReference_5x7.pdf. Accessed 6 Sept 2016

19. Eisenhauer EA, Therasse P, Bogaerts J et al (2009) New response evaluation criteria in solid tumours: revised RECIST guideline (version 1.1). Eur J Cancer 45:228-247

20. Tannir NM, Forero-Torres A, Ramchandren R et al (2014) Phase I dose-escalation study of SGN-75 in patients with CD70-positive relapsed/refractory non-Hodgkin lymphoma or metastatic renal cell carcinoma. Investig New Drugs 32:1246-1257

21. Moskowitz CH, Forero-Torres A, Shah BD et al (2014) Interim analysis of a phase 1 study of the antibody-drug conjugate SGNCD19A in relapsed or refractory B-lineage non-Hodgkin lymphoma. Blood 124:1741-1748

22. Fathi AT, Chen R, Trippett TM et al (2014) Interim analysis of a phase 1 study of the antibody-drug conjugate SGN-CD19A in relapsed or refractory B-lineage acute leukemia and highly aggressive lymphoma. Blood 124:963-969

23. Eaton JS, Miller PE, Mannis MJ et al (2015) Ocular adverse events associated with antibody-drug conjugates in human clinical trials. J Ocul Pharmacol Ther 31:589-604

24. Moore KN, Martin LP, Matulonis UA et al (2016) IMGN853 (mirvetuximab soravtansine), a folate receptor alpha (FR $\alpha)$ targeting antibody-drug conjugate (ADC): Single-agent activity in platinum-resistant epithelial ovarian cancer (EOC) patients (pts). J Clin Oncol 34 (suppl; abstr 5567)

25. Rudin CM, Pietanza MC, Bauer TM et al (2016) Safety and efficacy of single-agent rovalpituzumab tesirine (SC16LD6.5), a deltalike protein 3 (DLL3)-targeted antibody-drug conjugate (ADC) in recurrent or refractory small cell lung cancer (SCLC). J Clin Oncol 34 (suppl; abstr LBA8505)

26. Shor B, Kahler J, Dougher M et al (2016) Enhanced antitumor activity of an anti-5T4 antibody-drug conjugate in combination with PI3K/mTOR inhibitors or taxanes. Clin Cancer Res 22:383394

27. Shapiro G, Bell-McGuinn K, Molina J et al (2015) First-in-human study of PF-05212384 (PKI-587), a small-molecule, intravenous, dual inhibitor of PI3K and mTOR in patients with advanced cancer. Clin Cancer Res 21:1888-1895

28. Del Campo JM, Birrer M, Davis C et al (2016) A randomized phase II non-comparative study of PF-04691502 and gedatolisib (PF05212384) in patients with recurrent endometrial cancer. Gynecol Oncol 142:62-69

29. Damelin M, Zhong W, Myers J, Sapra P (2015) Evolving strategies for target selection for antibody-drug conjugates. Pharm Res 32: 3494-3507

30. Tian F, Lu Y, Manibusan A et al (2014) A general approach to sitespecific antibody drug conjugates. Proc Natl Acad Sci U S A 111: $1766-1771$ 